\title{
Analysis of a discrete-layout bimorph disk elements piezoelectric deformable mirror
}

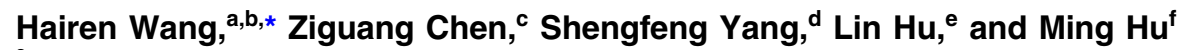 \\ ${ }^{a}$ Chinese Academy of Sciences, Purple Mountain Observatory, Nanjing, China \\ ${ }^{\mathrm{b} C h i n e s e}$ Academy of Sciences, Key Laboratory for Radio Astronomy, Nanjing, China \\ cHuazhong University of Science and Technology, Department of Mechanics, Wuhan, China \\ Indiana University-Purdue University, Purdue School of Engineering and Technology, Department of Mechanical and Energy Engineering, \\ Indianapolis, Indiana, United States \\ ${ }^{e}$ Chongqing Qianwei Science and Technology Group, Co., Ltd., Chongqing, China \\ ${ }^{\text {f}}$ Chinese Academy of Sciences, Institute of Geodesy and Geophysics, State Key Laboratory of Geodesy and Earth's Dynamics, Wuhan, China
}

\begin{abstract}
We introduce a discrete-layout bimorph disk elements piezoelectric deformable mirror (DBDEPDM), driven by the circular flexural-mode piezoelectric actuators. We formulated an electromechanical model for analyzing the performance of the new deformable mirror. As a numerical example, a 21-actuators DBDEPDM with an aperture of $165 \mathrm{~mm}$ was modeled. The presented results demonstrate that the DBDEPDM has a stroke larger than $10 \mu \mathrm{m}$ and the resonance frequency is $4.456 \mathrm{kHz}$. Compared with the conventional piezoelectric deformable mirrors, the DBDEPDM has a larger stroke, higher resonance frequency, and provides higher spatial resolution due to the circular shape of its actuators. Moreover, numerical simulations of influence functions on the model are provided. (c) 2018 Society of Photo-Optical Instrumentation Engineers (SPIE) [DOI: 10.11177/1.JATISI4.2.029001]
\end{abstract}

Keywords: deformable mirror; circular flexural-mode piezoelectric actuator; electromechanical model; higher resonance frequency; larger stroke.

Paper 17090 received Nov. 3, 2017; accepted for publication Apr. 23, 2018; published online May 10, 2018.

\section{Introduction}

The deformable mirror, usually driven by an array of actuators, is used as a key component in adaptive optics. 呕 The control of adaptive optics with real-time closed-loop fashion applied in adaptive optics has a cutoff frequency, which must be smaller than the resonance frequency of the deformable mirror ${ }^{\square}$ In addition to, the frequency range of atmospheric turbulence is from 0 to $10^{3} \mathrm{~Hz}$. The bandwidth of the adaptive optics system is mainly limited by the resonance frequency of the deformable mirror. ${ }^{-}$Recently, the adaptive optics telescopes have become larger and larger, and much more expensive, such as TMT and LOT ${ }^{\text {T }}$ These adaptive optics telescopes require a deformable mirror with large stroke, high resonance frequency but low cost. Two kinds of deformable mirrors, the conventional discrete-layout piezoelectric deformable mirror (CDPDM) and conventional bimorph piezoelectric deformable mirror (CBPDM), have been widely used in adaptive optics Q $^{\text {T }}$ The former is usually supported by an array of conventional piezoelectric actuators, coupled to a continuous mirrored face sheet. The other is a bimorph piezoelectric compound structure, made of a glass sheet bonded firmly to a piezoceramics layer with an appropriate electrode pattern on the back. The former has a higher temporal response, whereas the latter has a larger stroke with a lower cost.

The piezoelectric bimorph-beam-bending actuator and the circular flexural-mode piezoelectric actuator (CFPA), of different bimorph piezoelectric compound structures, have been analyzed in our previous works, and the results show that the bending

*Address all correspondence to: Hairen Wang, E-mail: Hairenwang@pmqaa cr actuators have larger strokes, higher resonance frequencies,

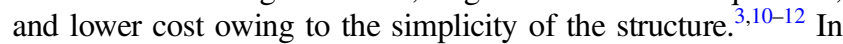
our previous work, the piezoelectric bimorph-beam-bending actuator was applied in a discrete-layout bimorph piezoelectric deformable mirror (DBPDM). Compared with the two kinds of conventional deformable mirrors mentioned above, the stroke of the DBPDM is larger than that of CDPDM and CBPDM; the resonant frequency of the DBPDM is higher than that of CBPDM and almost equal to that of CDPDM. Here, we propose a discretelayout bimorph disk elements piezoelectric deformable mirror (DBDEPDM) driven by the CFPA. Compared with the DBPDM, the new deformable mirror is highly controllable and provides higher spatial resolution due to the circular shape of the CFPA while maintaining the large stroke and the high resonance frequency.

\section{Electromechanical Model of the New Deformable Mirror}

The deformable mirror, supported by an array of CFPA, is coupled to a continuous mirrored face sheet (see Fig. 1). The glass sheet connects to the centers of CFPAs via circle push pads, which are identical cylinders with a radius $r_{\text {pad }}$ and a cross-section area $A_{\text {pad }}=\pi r_{\text {pad }}^{2}$. As shown in Figs. 2(a) 2(c), the simply supported CFPA consists of a metallic layer sandwiched by two identical piezoelectric ceramic layers. The piezoelectric layers are polarized in the $z$-direction. As indicated in Fig. 2(a), $2 h$ denotes the total thickness of the CFPA, $2 a\left(a \gg r_{\text {pad }}\right)$ denotes its diameter, and $2 c$ is the thickness of the metal layer. The top and bottom electrodes, parallel to each

$2329-4124 / 2018 / \$ 25.00$ (C) 2018 SPIE 


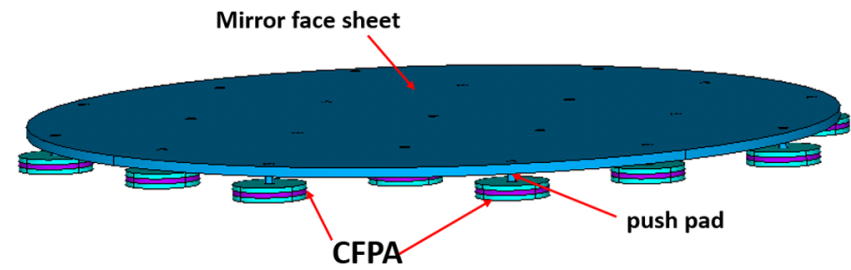

Fig. 1 The configuration of a deformable mirror driven by the CFPAs.

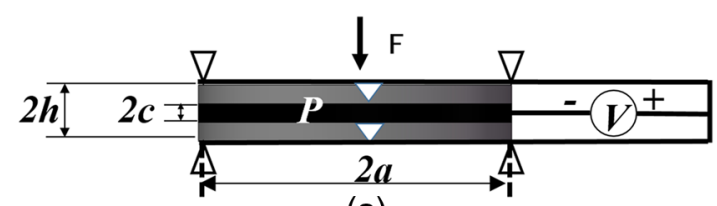

(a)

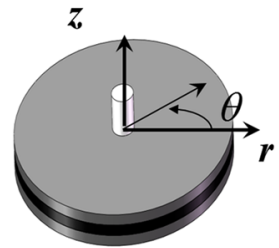

(b)

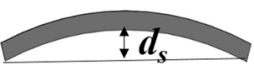

(c)
Fig. 2 (a) A simply supported CFPA with loading an external force, (b) a CFPA and its push pad, and (c) the dome height $d$.

other, have the same voltage across. The central metallic layer, functioned as the third electrode, is shared by the upper and lower piezoelectric layers. The actuator is simply supported at the outer boundary $(r=a)$, where any in-plane displacement is prohibited. Once a loading voltage $V$ is applied at the actuator, the upper flexible ceramic layer will extend or contract while the lower one will shrink, which generates a flexural deformation in the circular bimorph structure, as shown in Fig. 2(c). In the deformed configuration, the dome height, the distance from the horizontal plane to the center of the deformed plate, is referred to as the stroke $d_{\mathrm{s}}$, which is also the deflection at $r=0$. Here, $\left.d_{\mathrm{s}}\right|_{r=0}$ and $\left.d_{\mathrm{s}}\right|_{r=r_{\mathrm{pad}}}$ are approximately equal.

As the CFPA thickness is much smaller than its radius, CFPA can be treated as a thin plate. Moreover, the CFPA thickness is far larger than the dome height $d$ (when the CFPA is in operation). Therefore, the small deflection theory of thin plates is valid here. Accordingly, we have the following assumptions. 13.4

1. The vertical normal strain $S_{z}$ is very small and can be neglected, so $S_{z}=\partial u_{z} / \partial z \approx 0$, and $u_{z}=u_{z}(r, \theta)=$ $w$, where $w$ is the vertical displacement of the microelement on its middle plane.

2. The components of the stress, $T_{r z}, T_{\theta Z}$, and $T_{r \theta}$, are fairly smaller than other components of the stress, the displacements generated by these three components of the stress can be neglected, i.e., $T_{r z}=T_{\theta z}=T_{r \theta}=0$.

3. When a perpendicular force is applied on the thin plate, no displacement in the direction parallel to the plate is generated, i.e., $\left.u_{r}\right|_{z=0}=0,\left.u_{\theta}\right|_{z=0}=0$. Furthermore, this problem is axially symmetrical regardless of $\theta$, therefore, $u_{z}=u_{z}(r)=w$.
When the DBDEPDM is working, each CFPA is acted upon by external forces from other active CFPAs. In this paper, the resultant of external forces $F$ is defined by integrating the principal stress over the cross-section area of the push pad [see Fig. 2(a)]

$F=\int_{A} T_{z} \mathrm{~d} S=\bar{T}_{z} A_{\mathrm{pad}}=\pi r_{\mathrm{pad}}^{2} \bar{T}_{z}$

where $T_{z}$ denotes the principal stress of the cross-section area and $\bar{T}_{Z}$ denotes the average value of $T_{z}$. In the following, the analytical solutions of CFPA are derived in a cylindrical coordinate system. The $z$ direction is normal to the layers' surface, and the $r$ direction and the $\theta$ direction are the radial direction and the circumferential direction, respectively. Here, $(r, \theta, z)$ corresponds to $(1,2,3)$. According to the electrode configurations, the electric field in the actuator has the following components:

$E_{1}=0, E_{2}=0, E_{3}= \begin{cases}-V /(h-c), & \text { at } c<z<h, \\ V /(h-c) & \text { at }-h<z<-c .\end{cases}$

We consider the axis-symmetrically flexural deformation of the actuator. Here, $u_{z}(r)$ denotes the axially symmetrical deflection. With the assumption $2 h / a \ll 1$, the normal strains $S_{r}$ and $S_{\theta}$ of actuator can be expressed as $-z \partial^{2} u_{z} / \partial r^{2}$ and $-(z / r) \partial u_{z} / \partial r$, respectively $\$$ Because the plate is thin, $T_{23}$ and $T_{31}$ are much smaller than the in-plane principal stress components and are thus neglected. 10 The axis-symmetrically deflection requires $u_{\theta}=0$ and $\partial(\cdot) / \partial \theta=0$, which yields $T_{12}=0$. In light of the polarized direction and $c_{11}=c_{12}$, $c_{13}=c_{23}, e_{31}=e_{32}$, and the vanishing normal stress because the plate is thin, we obtain the constitutive relations of the top and the bottom piezoelectric plates as follows, respectively

$$
\begin{aligned}
& { }^{1} T_{r r}=-c_{11}^{p} z \partial^{2} u_{z} / \partial r^{2}-c_{12}^{p} z \partial u_{z} /(r \partial r)+e_{31}^{p} V /(h-c), \\
& { }^{1} T_{\theta \theta}=-c_{12}^{p} z \partial^{2} u_{z} / \partial r^{2}-c_{11}^{p} z \partial u_{z} /(r \partial r)+e_{31}^{p} V /(h-c), \\
& { }^{3} T_{r r}=-c_{11}^{p} z \partial^{2} u_{z} / \partial r^{2}-c_{12}^{p} z \partial u_{z} /(r \partial r)-e_{31}^{p} V /(h-c), \\
& { }^{3} T_{\theta \theta}=-c_{12}^{p} z \partial^{2} u_{z} / \partial r^{2}-c_{11}^{p} z \partial u_{z} /(r \partial r)-e_{31}^{p} V /(h-c),
\end{aligned}
$$

where $\left(c_{11}^{p}, c_{12}^{p}\right)=\left(c_{11}, c_{12}\right)-\left(c_{13}^{2}, c_{13}^{2}\right) / c_{33}, \quad e_{31}^{p}=e_{31}-$ $e_{33} c_{13} / c_{33}$. In addition, we also obtain the constitutive relations of the metal layer

$$
\begin{aligned}
& { }^{2} T_{r r}=-c_{11}^{A} z \partial^{2} u_{z} / \partial r^{2}-c_{12}^{A} z \partial u_{z} /(r \partial r), \\
& { }^{2} T_{\theta \theta}=-c_{12}^{A} z \partial^{2} u_{z} / \partial r^{2}-c_{11}^{A} z \partial u_{z} /(r \partial r),
\end{aligned}
$$

where $c_{12}^{A}=\nu E_{m} /[(1+\nu)(1-2 \nu)], c_{11}^{A}=c_{12}^{A}+E_{m} /[2(1-\nu)]$. $E_{m}$ and $\nu$ are the Young's modules and Poisson's ratio of the metal, respectively. By integrating the principal stress over the thickness direction of the plates, we obtain the bending moments per unit length. From the constitutive relations of the layers, we obtain 


$$
\begin{aligned}
& M_{r}=\int_{-h}^{h} T_{r r} z \mathrm{~d} z=-\frac{2 c^{3}}{3}\left(B_{1} \frac{\partial^{2} u_{z}}{\partial r^{2}}+B_{2} \frac{\partial u_{z}}{r \partial r}\right)+e_{31}^{p} V(h+c), \\
& M_{\theta}=\int_{-h}^{h} T_{\theta \theta} z \mathrm{~d} z=-\frac{2 c^{3}}{3}\left(B_{2} \frac{\partial^{2} u_{z}}{\partial r^{2}}+B_{1} \frac{\partial u_{z}}{r \partial r}\right)+e_{31}^{p} V(h+c),
\end{aligned}
$$

with $\left(B_{1}, B_{2}\right)=\left(c_{11}^{A}+\gamma c_{11}^{p}, c_{12}^{A}+\gamma c_{12}^{p}\right)$ and $\gamma=(h / c)^{3}-1$. The transverse shear force per unit length is obtained by the following integral of the shear stress over the dimension $z$ of the actuator 13

$$
\begin{aligned}
Q_{r} & =\int_{-h}^{h} T_{r z} \mathrm{~d} z=\partial M_{r} / \partial r+\left(M_{r}-M_{\theta}\right) / r \\
& =-\left(2 c^{3} B_{1} / 3\right)\left(\frac{\partial^{3} u_{z}}{\partial r^{3}}+\frac{\partial^{2} u_{z}}{r \partial r^{2}}-\frac{\partial u_{z}}{r^{2} \partial r}\right) .
\end{aligned}
$$

The equation of equilibrium in a slim plate takes the following form: 13

$$
Q_{r} / r+\partial Q_{r} / \partial r=0
$$

The equation of equilibrium in a circular thin plate has the following form: 묘

$\nabla^{2} \nabla^{2} u_{z}=0$

where $u_{z}(r)$ denotes the axially symmetrical deflection and $\nabla^{2}$ is the Laplacian. As the bimorph piezoelectric circular actuator is simply supported at $r=a$, we have obtained

$$
\left.\frac{\partial u_{z}}{\partial r}\right|_{r=r_{\mathrm{pad}}}=0,2 \pi r_{\mathrm{pad}} Q_{r}\left(r_{\mathrm{pad}}\right)=F, M_{r}(a)=0, u_{z}(a)=0,
$$

where $Q_{r}$ denotes the transverse shear force per unit length, and $M_{r}$ is the bending moment of the $r$-direction. The specific equations of $Q_{r}$ and $M_{r}$ can be found in Refs. 11] and 14. The general solution to Eq. (9) can be written as

$u_{z}=W_{1}+W_{2} r^{2}+W_{3} \log r+W_{4} r^{2} \log r$,

where $W_{1}-W_{4}$ are constants to be determined. From Eqs. (6), (7), (10), and (11), we can obtain as

$$
\begin{aligned}
& 2 W_{2} r_{\mathrm{pad}}+W_{3} / r_{\mathrm{pad}}+W_{4}+2 W_{4} r_{\mathrm{pad}} \log r_{\mathrm{pad}}=0, \\
& -\frac{4}{3} B_{1} c^{3} \pi\left(2 W_{2}+W_{3} / r_{\mathrm{pad}}^{2}+5 W_{4}+2 W_{4} \log r_{\mathrm{rad}}\right)=F,
\end{aligned}
$$

$$
\begin{gathered}
e_{31}^{p}(c+h) V-\frac{2}{3} c^{3}\left[B_{1}\left(2 W_{2}-W_{3} / a+3 W_{4}+2 W_{4} \log a\right)\right. \\
\left.+B_{2}\left(2 W_{2}+W_{3} / a^{2}+W_{4}+2 W_{4} \log a\right)\right]=0,
\end{gathered}
$$

$$
W_{1}+W_{2} a^{2}+W_{3} \log a+W_{4} a^{2} \log a=0 .
$$

By solving Eqs. (12)-(15), we can obtain

$$
\begin{aligned}
& W_{1}=\Lambda_{1} F+\Lambda_{2} V, \\
& W_{2}=\Lambda_{3} F+\Lambda_{4} V, \\
& W_{3}=\Lambda_{5} F+\Lambda_{6} V, \\
& W_{4}=\Lambda_{7} F,
\end{aligned}
$$

where

$$
\begin{aligned}
\Lambda_{1}= & a^{2}\left\{-a^{2}\left(3 B_{1}+B_{2}\right)+\left(B_{2}-B_{1}\right) r_{\mathrm{pad}}^{2}\right. \\
& +2 r_{\mathrm{pad}}^{2}\left\{2\left(B_{1}+B_{2}\right) \log ^{2} a\right. \\
& +\left(B_{2}-B_{1}\right) \log r_{\mathrm{pad}}-\log a\left[B_{2}-3 B_{1}\right. \\
& \left.\left.\left.+2\left(B_{1}+B_{2}\right) \log r_{\mathrm{pad}}\right]\right\}\right\} / \Gamma_{2} . \\
\Lambda_{2}= & -3 a^{2} e_{31}^{p}(c+h)\left(a^{2}-r_{\mathrm{pad}}^{2} \log a\right) / \Gamma_{1}, \\
\Lambda_{3}= & -a^{2}\left(3 B_{1}+B_{2}\right)+\left(B_{2}-B_{1}\right) r_{\mathrm{pad}}^{2} \\
& \left.-2 a^{2}\left(B_{1}+B_{2}\right) \log a+2\left(B_{2}-B_{1}\right) r_{\mathrm{pad}}^{2} \log r_{\mathrm{pad}}\right] / \Gamma_{1}, \\
\Lambda_{4}= & 3 a^{2} e_{31}^{p}(c+h) / \Gamma_{2}, \\
\Lambda_{5}= & -4 a r_{\mathrm{pad}}^{2}\left[B_{1}+\left(B_{1}+B_{2}\right)\left(\log a-\log r_{\mathrm{pad}}\right)\right] / \Gamma_{1}, \\
\Lambda_{6}= & -6 a^{2} e_{31}^{p}(c+h) r_{\mathrm{pad}}^{2}, \\
\Lambda_{7}= & -1 /\left(8 B_{1} c^{3} \pi\right), \\
\Gamma_{1}= & 16 B_{1} c^{3} \pi\left[a^{2}\left(B_{1}+B_{2}\right)+\left(B_{1}-B_{2}\right) r_{\mathrm{pad}}^{2}\right], \\
\Gamma_{2}= & 16 B_{1}\left(B_{1}+B_{2}\right) c^{3} \pi .
\end{aligned}
$$

$\Lambda_{1}-\Lambda_{7}$ are constants and depend on the geometric parameters $\left(a, c, h, r_{\text {pad }}\right)$ and material parameters of the piezoelectric ceramic layer and the central metallic layer. Thus, we can obtain

$$
\begin{aligned}
d_{s}= & u_{z}\left(r_{\mathrm{pad}}\right)=\Lambda_{1} F+\Lambda_{2} V+\left(\Lambda_{3} F+\Lambda_{4} V\right) r_{\mathrm{pad}}^{2} \\
& +\left(\Lambda_{5} F+\Lambda_{6} V\right) \log r_{\mathrm{pad}}+\Lambda_{7} F r_{\mathrm{pad}}^{2} \log r_{\mathrm{pad}}
\end{aligned}
$$

where $d_{s}$ denotes the dome height at $r=r_{\text {pad }}$, which is equal to the stroke of the DBDEPDM. From Eq. (18), we have obtained

$$
\begin{aligned}
& F=k_{V} V+k_{s} d_{s}, \quad k_{V}=-\left(\Lambda_{2}+\Lambda_{4} r_{\mathrm{pad}}^{2}+\Lambda_{6} \log r_{\mathrm{pad}}\right) k_{s}, \\
& k_{s}=1 /\left(\Lambda_{1}+\Lambda_{3} r_{\mathrm{pad}}^{2}+\Lambda_{5} \log r_{\mathrm{pad}}+\Lambda_{7} r_{\mathrm{pad}}^{2} \log r_{\mathrm{pad}}\right) .
\end{aligned}
$$

The CFPA at different sites of DBDEPDM drives the continuous mirrored surface of DBDEPDM. The continuous mirrored glass sheet can be seen as a linear elastic structure, which impedes the motion of the CFPA array. The number of

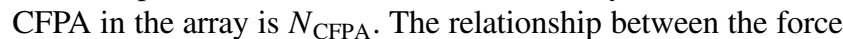
and the displacement for the face sheet within the CFPA array is

$\left\{F^{J}\right\}=\left[K_{\mathrm{dm}}^{M J}\right]\left\{d_{s}^{J}-d_{a}\right\}$,

$K_{\text {dm }}$ denotes the $N_{\text {CFPA }}$ by $N_{\text {CFPA }}$ stiffness matrix of the continuous mirrored glass sheet, and $F$ and $d$ are vectors with the $N_{\text {CFPA }}$ components. $d^{J}$ denotes the stroke of the $J$ 'th CFPA, and $F^{J}$ denotes the corresponding force at the $J$ 'th CFPA location. 
$d_{a}$ denotes the average displacement of all the locations of the CFPAs of DBDEPDM.

We computed $K_{\mathrm{dm}}$ by the finite element analysis. Taking a deformable mirror of 21 CFPAs as an example [see Fig. 4(a)], we created a finite element model by only including the face sheet and the push pads, as shown in Fig. 4(a). The CFPAs were not involved in the finite element model of Fig. 4(a). The push pads and the face sheet were modeled in three dimensions. The CFPA locations of the stiffness matrix of the face sheet were computed when giving a normal unit displacement at one CFPA location (the $J$ 'th) and keeping the other CFPA displacements zero. The force results at the CFPA locations are the stiffness matrix's $J$ 'th row. We can obtain the whole matrix by the computation repeated at each actuator location. The resultant $K_{\mathrm{dm}}$ is symmetric due to Betti's reciprocal theorem for linear elastic problems. Using Eqs. (19) and (20), we have obtained

$$
\left[K_{\mathrm{dm}}^{M N}\right]\left\{d^{J}-d_{a}\right\}=k_{s}\left\{d^{J}\right\}+k_{v}\left\{V^{J}\right\} .
$$

In Eq. (21), $k_{s}$ and $k_{v}$, provided by Eq. (19), depend on the material and geometric parameters of the CFPAs. In this paper, we assume that all the CFPAs are identical. $k_{s}$ and $k_{v}$ are the same for different sites of the deformable mirror. In the future work, the CFPAs at different sites can be designed with different parameters to achieve a higher spatial resolution, and $k_{s}$ and $k_{v}$ can have various values. The electrical interconnects, which may be another factor limiting the actuator density, will be addressed in our future work. The average displacement for the actuator array is $d_{a}=\left(1 / N_{\mathrm{CFPA}}\right) \sum_{J} d^{J}$. From Eq. (21), we have obtained

$$
[K]\left\{d^{J}\right\}=k_{v}\left\{V^{J}\right\} .
$$

The details of $[K]$ see Ref. 2 . Finally, the normal displacement $w(x, y)$ in the mirror face sheet is reconstructed with the influence functions $f^{J}(x, y)$ using

$$
w(x, y)=\sum_{J} f^{J}(x, y) d^{J}
$$

\section{Numerical Results and Discussion}

In this paper, the piezoelectric material of the CFPA is PZT-5H, and its parameters are listed below

$$
\begin{aligned}
\left(c_{11}, c_{12}, c_{13}, c_{33}\right) & =(12.6,7.95,8.41,11.7) \times 10^{10} \mathrm{~N} / \mathrm{m}^{2}, \\
\left(e_{31}, e_{33}\right) & =(-6.5,23.3) \mathrm{C} / \mathrm{m}^{2}
\end{aligned}
$$

The geometric parameters of the CFPA are listed below: $a=15 \mathrm{~mm}, \quad \Delta h=h-c=1 \mathrm{~mm}, \quad \eta=c / \Delta h=1 / 5, \quad$ and $r_{\text {pad }}=2 \mathrm{~mm}$. The metal layer of the CFPA is aluminum alloy (Al) with the Poisson's ration $\nu=0.33$ and the Young's modulus $E_{m}=70 \mathrm{GPa}$. The constants $\Lambda_{1}-\Lambda_{7}$ in Eqs. (18) and (19) depend on the above material parameters and the geometric parameters.

Figure 3(a) shows the relationships between $d_{s}$ and the electric field at different stresses. From Fig. B(a), it should be observed that $d_{s}$ has a bias value caused by the external force. The value of the bias is due to the effectively concentrated force. If the bias value is positive at $\bar{T}_{z}>0$, it means that the effective concentrated force is a tensile force. Conversely, if the bias value is negative at $\bar{T}_{z}<0$, it indicates that the effectively concentrated force is a compression force. We can observe from Fig. 3(a) that the stroke values of the CFPA are larger than $10 \mu \mathrm{m}$ at about $\left|E_{3}\right|=0.5 \mathrm{MV} / \mathrm{m}$, and the strokes equal to the strokes of DBDEPDM [see Eqs. (19) and (21)]. Therefore, the results have suggested the new deformable mirror's capability to have a large stroke. Figure 3(b) shows the dependence of $d_{s}$ upon the radius $a$ of CFPA for $\bar{T}_{z}=0$ and electric field $=0.5 \mathrm{MV} / \mathrm{m}$. It is clearly observed that the relationship between $d_{\mathrm{s}}$ and the radius $a$ is nonlinear, and $d_{\mathrm{s}}$ increases with the increasing radius $a$. The DBDEPDM can be easier to provide a high spatial resolution with more CFPAs having a smaller radius $a$, while keeping its stroke still larger than $10 \mu \mathrm{m}$.

Here, in the finite element model of Fig. 4(a), we used the single-crystal silicon with Young's modulus $E_{\mathrm{si}}=190 \mathrm{GPa}$ and Poisson's ration $\nu=0.3$ as the material of pads and the face sheet. The radius of the mirror face sheet is $R_{0}=82.5 \mathrm{~mm}$, and the thickness of the mirror is $h_{0}=2 \mathrm{~mm}$. With the electromechanical model, the influence functions of all CFPAs sites of the deformable mirror can be obtained. For example, only the No. 1 CFPA [the site is marked by 1 in Fig. 4(b)] was excited by the applied voltage $1 \mathrm{~V}$, and other CFPAs, whose voltages are all equal to $0 \mathrm{~V}$, were not excited. Then a voltage vector $\left\{V^{J}\right\}$ was created. In this case, only the effectively concentrated force of No. 1 CFPA is a compression force, whereas the effective concentrated forces of the other CFPAs are all tensile forces. Inserting $\left\{V^{J}\right\}$ into Eq. (22), we can obtain the displacement vector $\left\{d^{J}\right\}=k_{v}[K]^{-1}\left\{V^{J}\right\}$, which serves as the input

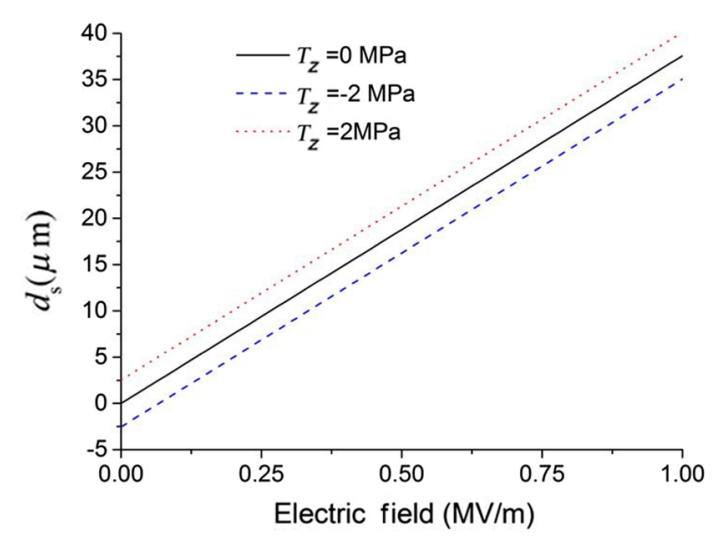

(a)

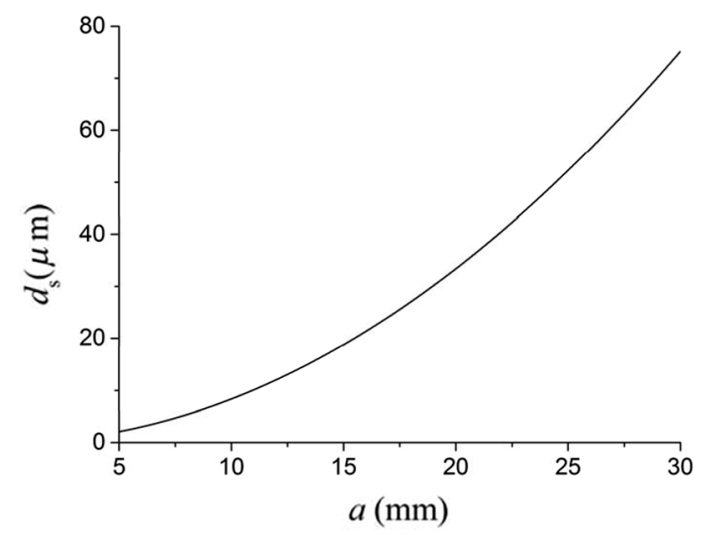

(b)

Fig. 3 (a) The dependence of $d_{\mathrm{s}}$ upon the electric field for different stresses of $A_{\text {pad. }}$. (b) The dome height $d_{s}$ versus the radius a for $\left|E_{3}\right|=0.5 \mathrm{MV} / \mathrm{m}$. 


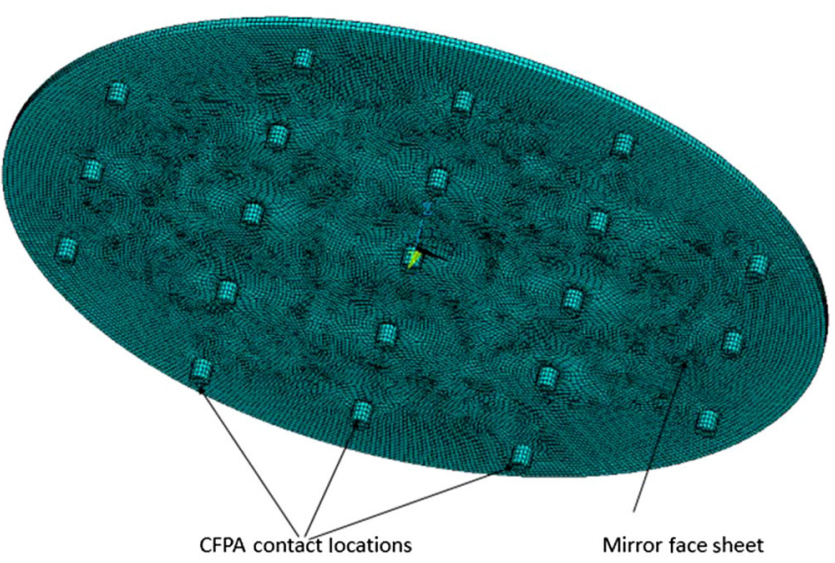

(a)

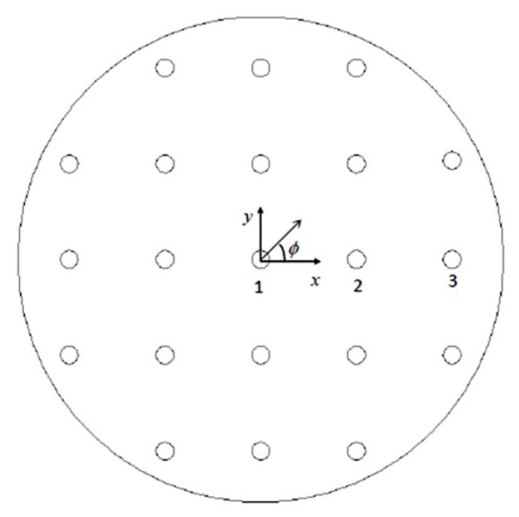

(b)

Fig. 4 The mirror face sheet and pads of a deformable mirror of 21 elements. (a) The 3-D finite element analysis model of mirror face sheet and push pads and (b) the corresponding coordinate system and sites of Nos. 1, 2, and 3 CFPAs.

displacement loads to be applied to the bottoms of the circular pads of the finite element model [see Fig. 4(a)]. After calculation, the resulting displacement field in the surface of the face sheet has been used to define the influence function $f^{J}(x, y)$. Hence, we can obtain the three-dimensional (3-D) influence function of the face sheet surface surrounding No. 1 CFPA [see Fig. 5(a)]. With the same way, we can obtain influence functions of No. 2 CFPA and No. 3 CFPA. Figure 5(b) shows the two-dimensional (2-D) influence functions of No. 1 CFPA, No. 2 CFPA, and No. 3 CFPA, respectively. The influence functions of the other sites can also be obtained in the same way. Once the influence functions of the 21 sites have been obtained, the realistic electromechanical model could be used to simulate the correction of the wavefront errors of the adaptive optics system.

On the basis of the finite element model of Fig. 4(a), we added the CFPAs array to the model, and hence the finite element model of DBDEPDM driven by 21 CFPAs was obtained, as shown in Fig. 6. With the modal analysis, we obtained its first natural frequency of $4.456 \mathrm{kHz}$, which is much higher than $1.0 \mathrm{kHz}$ (the frequency range of atmospheric turbulence is from 0 to $10^{3} \mathrm{~Hz}$ ). When the CFPAs do not have the metallic layer, the first natural frequency of the deformable mirror is $3.652 \mathrm{kHz}$, which is lower than $4.456 \mathrm{kHz}$ but also higher than $1.0 \mathrm{kHz}$. Therefore, we can come to the conclusion

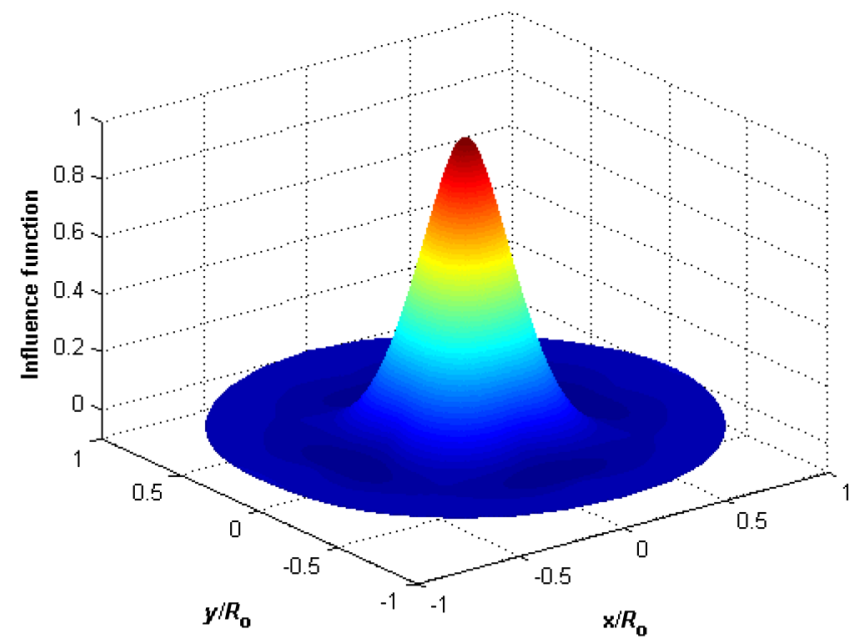

(a)

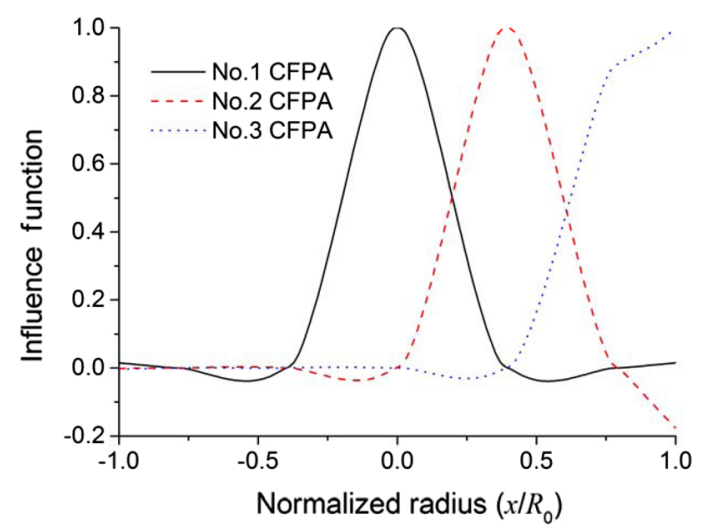

(b)

Fig. 5 (a) The 3-D influence function for a unit voltage of the No. 1 site and (b) the 2-D influence functions for a unit voltage of the No. 1 site, No. 2 site, and No. 3 site.

that DBDEPDM has a high natural frequency, and its natural frequency could become much higher by the addition of a metallic layer into the CFPAs.

Moreover, the quantitative comparisons between the two kinds of conventional PZT DMs have been presented. An FEA model of a CDPDM, which also has 21 elements and was modeled on a DM in Ref. 15, is shown in Fig. 7. Here, the size and material of continuous mirrored face sheet of the CDPDM are same to those of the DBDEPDM, and the size and material of actuators of the CDPDM are same to those of the DM in Ref. 15. With the modal analysis, we obtained the CDPDM's first natural frequency of $6.600 \mathrm{kHz}$, which is also larger than $1.0 \mathrm{kHz}$ (the frequency range of atmospheric turbulence is from 0 to $10^{3} \mathrm{~Hz}$ ). However, from Ref. 15, we can see that the stroke of the actuators of the CDPDM is smaller than $4 \mu \mathrm{m}$, which is much smaller than the stroke of the DBDEPDM.

An FEA model of a CBPDM, which also has 21 elements, is shown in Fig. 8 Here, the size and material of continuous mirrored face sheet of the CBPDM are also same to those of the DBDEPDM, the thickness of PZT layer of CBPDM equals to the total thickness of two layer of PZT element of DBDEPDM. As well known, the CBPDM has a large stroke. However, with the modal analysis, we obtained the 


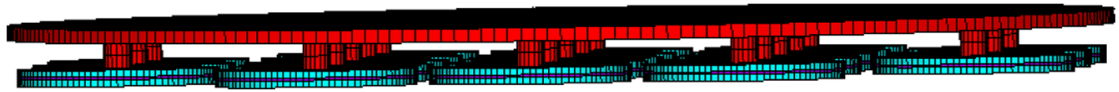

Fig. 6 The resonance frequency of the whole DBDEPDM is gained with the mode analysis of whole finite element model, whose resonant frequency much higher than $1.0 \mathrm{KHz}$.

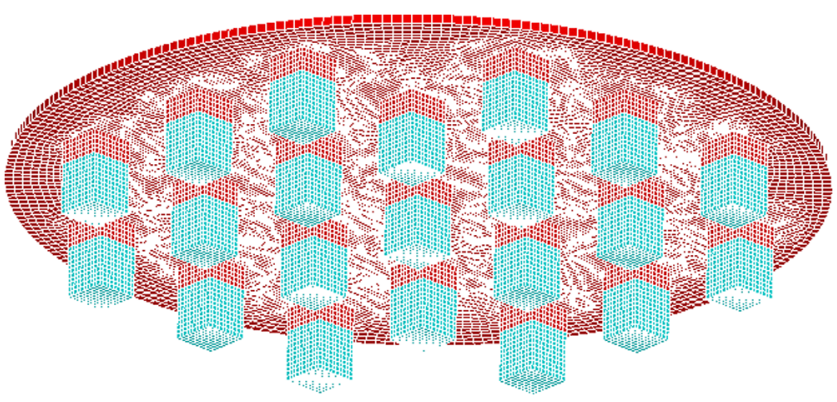

Fig. 7 An FEA model of a CDPDM.

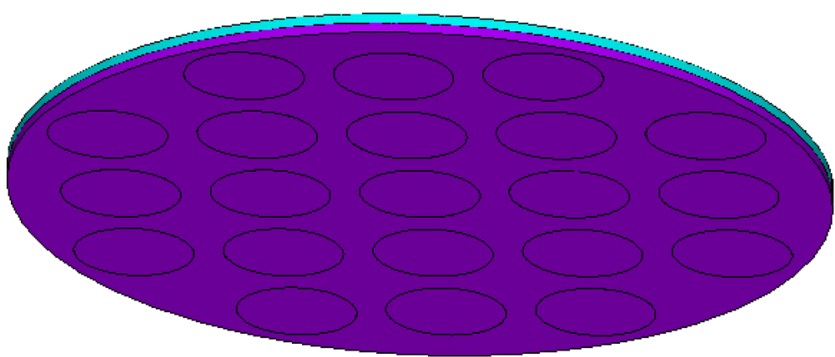

Fig. 8 An FEA model of a CBPDM.

CBPDM's first natural frequency of $0.684 \mathrm{kHz}$, which is smaller than $1.0 \mathrm{kHz}$ (the frequency range of atmospheric turbulence is from 0 to $10^{3} \mathrm{~Hz}$ ) and far less than that of the DBDEPDM $(4.456 \mathrm{kHz})$.

Therefore, the DBDEPDM has both a larger stoke range and higher resonant frequencies comparing with the two kinds of conventional PZT DMs.

\section{Summary}

A continuous mirrored deformable mirror has been analyzed. The simulation results suggest that in addition to having a larger stroke and a higher resonance frequency, the deformable mirror is also easier to provide a high spatial resolution due to the circular shape of the CFPA. Furthermore, numerical simulations of influence functions in the model have been successfully performed.

\section{Acknowledgments}

This work has been supported by the National Natural Science Foundation of China (Grant Nos. 11403109, 11190014, and 11373073) and the Natural Science Foundation of Jiangsu Province (Grant No. BK20141042). This work also has been supported by $2.5 \mathrm{~m}$ Wide Field Survey Telescope (WFST) project of China, the project has been started.

\section{References}

1. R. Tyson, Principles of Adaptive Optics, CRC Press, Boca Raton, Florida (2010).
2. H. Wang, "Research on a bimorph piezoelectric deformable mirror for adaptive optics in optical telescope," Opt. Express 25(7), 8115-8122 (2017).

3. H. Wang, "Analytical analysis of a beam flexural-mode piezoelectric actuator for deformable mirrors," T. Astron. Telesc. Instrum. Syst. 1(4), 049001 (2015).

4. B. Macintosh et al., "Extreme adaptive optics for the Thirty Meter Telescope," Proc. SPIE 6272, 62720N (2006).

5. D.-Q. Su et al., "A suggested 12-m telescope optical system configuration for China," Mon. Not. R. Astron. Soc. 460(2), 2286-2295 (2016).

6. P.-Y. Madec, "Overview of deformable mirror technologies for adaptive optics and astronomy," Proc. SPIE 8447, 844705 (2012).

7. C. Radzewicz et al., "Piezo-driven deformable mirror for femtosecond pulse shaping," Opt. Lett. 29(2), 177-179 (2004).

8. H. Wang et al., "Fast optimization of a bimorph mirror using x-ray grating interferometry," Opt. Lett. 39(8), 2518-2521 (2014).

9. R. Li et al., "Controllable continuous-wave $\mathrm{Nd}: \mathrm{YVO}_{4}$ self-Raman lasers using intracavity adaptive optics," Opt. Lett.39(16), 4762-4765 (2014).

10. H. Wang, "Linear and nonlinear analysis of the thermal effects of beam piezoelectric bending actuator on adaptive optics," T. Intell. Mater. Syst. Struct. 28(20), 3016-3024 (2017).

11. H. Wang and S. Yang, "Modeling and analysis of the thermal effects of a circular bimorph piezoelectric actuator," Appl. Opt. 55(4), 873-878 (2016).

12. H. Wang, H. Ming, and L. Zhengqin, "Modelling and analysis of circular bimorph piezoelectric actuator for deformable mirror," Appl. Math. Mech. 37(5), 639-646 (2016).

13. J. Wang et al., "On the strain-gradient effects in micro piezoelectricbimorph circular plate power harvesters," Smart Mater. Struct. 21(1), 015006 (2012).

14. H. R. Wang, Y. Hu, and J. Wang, "On the nonlinear behavior of a multilayer circular piezoelectric plate-like transformer operating near resonance," EEEE Trans. Ottrason. Ferroelectr. Freq. Contro 60(4), 752-757 (2013).

15. H. Craig, P. D. Dean, and S. R. Winzer, "Simulating electrostrictive deformable mirrors: I. Nonlinear static analysis," Smart Mater. Struct. 8(5), 691-699 (1999).

Hairen Wang received his ME degree in physics in 2009 and his PhD in mechanics from Huazhong University of Science and Technology (HUST), Wuhan, China, in 2013. Currently, he is an associate professor in Purple Mountain Observatory, Chinese Academy of Sciences. Now, he is taking part in 5-m Terahertz Telescope on Dome A (DATE5) design study and 2.5-m Wide Field Survey Telescope (WFST) of China design study. His research interests include deformable mirror fabrication and design, telescope's structural mechanics, and its thermal control system. He is a peer reviewer for Optics Express and Applied Optics.

Ziguang Chen received his $\mathrm{ME}$ degree in engineering mechanics from Central South University and his $\mathrm{PhD}$ in mechanical engineering and applied mechanics from the University of Nebraska-Lincoln, in 2007 and 2012, respectively. He is an associate professor in the Department of Mechanics, at Huazhong University of Science and Technology. He has authored over 30 peer-reviewed journal articles on piezoelectric devices design, computational modeling of materia degradation and structural failure, and surface/interface selfassembly. His current research interests include modeling and detection of stress-corrosion cracking, piezoelectric composites, and design of piezoelectric devices.

Shengfeng Yang received his $\mathrm{ME}$ degree in mechanics from Huazhong University of Science and Technology in 2010 and his $\mathrm{PhD}$ in mechanical engineering from the University of Florida in 2014. Currently, he is an assistant professor in the Department of Mechanical and Energy Engineering at Indiana University-Purdue 
University Indianapolis (IUPUI). His research interests include computational mechanics, multiscale-multiphysics modeling, and scientific computing with a particular focus on the thermo-mechanical and dynamical properties of materials including metallic alloys, ceramics, biological composite, and energy conversion and battery materials.

Lin Hu received his BE degree in mechanical engineering from Jilin University, Changchun, China, in 2005 and his ME degree in mechanics from Huazhong University of Science and Technology (HUST), Wuhan, China, in 2013. Currently, he is a mechanical engineer in
Chongqing Qianwei Science and Technology Group Co. Ltd. His research interests include piezoelectric devices design, deformable mirror design, and equipment mechanical structure design.

Ming Hu received his bachelor's degree in automation and doctor degree in physics from Huazhong University of Science and Technology (HUST), Wuhan, China. Currently, he is an associate researcher in Institute of Geodesy and Geophysics, CAS. His research interests include automatic control of deformable mirror and inertial sensors design. 